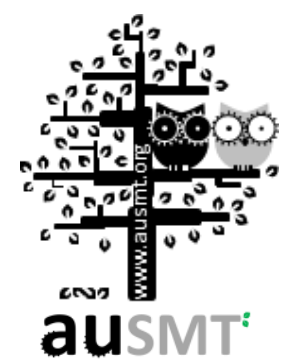

\title{
Fall Detection FPGA-Based Systems: A
} Survey

\section{Sahar Abdelhedi1,*, Riad Bourguiba1, Jaouhar Mouine ${ }^{2}$, Mouna Baklouti ${ }^{3,4}$, and Anis Youssef 4}

${ }^{1}$ Université de Tunis El Manar, Ecole Nationale d'Ingénieurs de Tunis, LR11ES20 Laboratoire d'Analyse, Conception et Commande des Systèmes, 1002, Tunis, Tunisia.

${ }^{2}$ Department of Electrical Engineering, Prince Sattam Bin Abdulaziz University Al-Kharj, Kingdom of Saudi Arabia

${ }^{3}$ Université de Sfax, Ecole Nationale d'Ingénieurs de Sfax, CES Lab, Sfax, Tunisia.

${ }^{4}$ TELNET Innovation Labs, TELNET Group, Parc Technologique El Ghazala, Tunisia

(Received 1 February 2016; Accepted 26 May 2016; Published on line 1 December 2016)

*Corresponding author: abdelhedi.sahar@gmail.com

DOI: 10.5875 /ausmt.v6i4.1105

Abstract: Falls can lead to serious injuries, hospitalization and sometimes death, and are considered the number one cause of disabilities among elderly people, making falls a key concern in the healthcare sector. Advances in medical technology and healthcare mechanisms have driven the development of new responses to the healthcare needs of a growing elderly population. Ambulatory accelerometer devices have been applied to develop reliable and robust fall detection systems. This paper assesses fall detection systems using Field Programmable Gate Arrays (FPGAs) as a CPU in addition to data transmission. In this paper, we give a survey of the different fall detection systems based on FPGAs in the literature, definition of the main theoretical points of fall detection accelerometers-based systems, existing techniques and algorithms and we give an overview of the main steps to design a fall detection system.

Keywords: accelerometers, embedded systems, fall detection, FPGA, health-monitoring

\section{Introduction}

The global elderly population is expected to grow dramatically over the coming decades. Falls are a major health hazard for older people, and are a key cause of trauma, disability, hospitalization and morbidity. Among elderly people, 55 percent of falls occur in the home, while another 23 percent occur in outdoor areas surrounding the home [1].

Wireless networks [2] and mobile computing devices allow for the development of new approaches to fall prevention and response. Portable devices originally developed for monitoring the health of chronically ill patients have been used to detect falls through the use of low-cost MEMs accelerometers [3]. A new trend in fall detection is the development of small, smart wearable devices with adaptive fall detection algorithms. Multiples technologies like micro-controllers [4], FPGAs [5] and smart phones [6] have been successfully used to detect falls. This survey mainly focuses on fall detection systems using FPGAs as a flexible accelerometer-based platform. The remainder of this paper is organized as follows. Section II reviews the theoretical background of fall detection. Section III provides a review of the literature on fall detection systems. Section IV describes and compares different FPGA-based fall detection systems. Section $V$ surveys existing techniques and algorithms for fall detection systems. Concluding remarks are provided in section VI.

\section{Background}

Falls by elderly people are a significant problem with an estimated $30 \%$ of older persons over 65 falling each year [7]. Various fall detection devices have been developed to allow elderly or chronically ill people to live safely and independently at home. 
Fall detection classification

A fall detection system is defined as a device that sends out an alert in response to a fall. A miniaturized fall detection device seeks to improve fall detection accuracy while having a minimal impact on the user's daily life. To this end, several attempts [8] [9] have been made to develop a variety of fall detection methods as follows (Fig.1):

\section{Vision based approach}

This approach uses fixed cameras that monitor all the patient's movements. The recorder video data are then forwarded to a CPU that detects falls following a

Sahar Abdelhedi received the MS degree in Information Technology from University Paris Descartes, Paris, France in 2012. She is currently pursuing the PhD in Microelectronics at the National School of Engineers of Tunis (ENIT), Tunis, Tunisia. From 2013, she is a PhD student working on health monitoring thematic, embedded systems and FPGA technology in collaboration with TELNET innovation department. Her research interest includes the design of a fall detection device implemented on an FPGA for elderly people.

Riad Bourguiba received the the PhD in run-time reconfigurable FPGA architectures from the National of School of Electronics and its applications (ENSEA), Paris, France in 2000. He is currently an Assistant Professor in the Electrical Department of the National School of Engineers of Tunis (ENIT). His researches deal with architecture design, embedded systems, systems-onchip, FPGAs and real-time systems.

Jaouhar Mouine received the PhD in electrical engineering from the University of Sherbrook, Sherbrook, Canada in 1992. From 2001 to 2004, he was a full Professor in the Electrical and Computer Science department of the University of Sherbrook, Sherbrook, Canada. From 2004 to 2015, he was a professor in the Electrical Department of the National School of Engineers of Tunis (ENIT). He is currently a full time professor in the Electrical Engineering Department of Prince Sattam Ibn Abdulaziz University, Al-Kharj, Saudi Arabia. He is the author of more than 60 papers in international journals and conferences and one of the inventors of the programmable neuro-simulator. His research interests includes nanotechnology, analog devices, 3D SoC design and Biomedical engineering. Pr Mouine was a lecturer of IEEE International Conference on Signal, Systems Devices from 2006 to 2010 and reveived the national decoration of merit in 2002 from the Tunisian government.

Mouna Baklouti received the engineering and M.S. degrees from the Tunisian Polytechnic School, Tunis, Tunisia in 2006 and 2007, respectively. She received a Ph.D in Computer Science from the National Engineering School of Sfax, Sfax, Tunisia and University of Lille 1, Lille, France in December 2010. She is is currently an Assistant Professor at the National Engineering School of Sfax, Tunisia. Her research interests include hardware/software co-design, massively parallel systems design and System-on-Chip design.

Anis Youssef received the Engineer degree from the National School of Engineers of Monastir (ENIM), Monastir, Tunisia in 2000, the master degree from the University Henri Poincaré of Nancy in 2001, Nancy, France and finally he received the PhD in Computer Science from the Institut National Polytechnique de Toulouse in 2005, Toulouse, France. He is currently Research and Innovation Activities Director at TELNET group. From June 2005 to December 2008, he joined the Department of Electrical Engineering and Electronic Systems (DIESE) at Renault technology center in Guyancourt, France. He was responsible for the design, development and industrialization of a function that handles the Features Activation Logic (FAL) and the aftercrash management function. He also coordinated with the R\&D service for the development of prototypes for electric and hybrid vehicles and to innovate in the field of passive security

From January 2009 to March 2010, he was a head of railway dependability project in ANSALDO, for the rail transport system in Sweden Banverket. He was responsible for the design, development and industrialization of a function that handles the Features Activation Logic (FAL) and the after-crash management function. specific fall pattern recognition algorithm. If a fall is detected, an alarm is triggered automatically [10]. This approach allows for more events to be detected simultaneously, making it less intrusive in the patient's daily life, and the acquired data can also be used for remote verification. Limitations include relatively high cost and implementation complexity, along with privacy concerns. Moreover, a fall can be detected only inside the home in rooms equipped with cameras.

\section{Environmental approach}

This approach installs infrared or pressure sensors in places where the patient is to be monitored for falls [11]. This approach uses cheap and non-intrusive devices, but installation is labor-intensive and fall detection is limited to areas with sensors.

\section{Wearable approach}

In this approach, the user wears sensors including gyroscopes and accelerometers collecting and transmitting data to the processing platform via wireless communications [12]. As the devices must be worn all the day, this approach is relatively more intrusive, but offers advantages in terms of cost, size, weight, power consumption, ease of use and portability. Our survey focuses on this approach. More precisely, we only consider systems that use the analysis of motion data for the detection of falls.

Wearable sensor-based systems

These systems can be worn by the subject during normal daily life to monitor location-specific (i.e., indoor or outdoor) physiological data. This provides a good alternative for systems which rely on human activity recognition [13]. Longitudinal data are collected through wearable sensors that can be integrated into clothing or worn as wearable devices. As they are attached to the patient and don't interact with the environment, wearable sensors can measure physiological parameters which may not be measurable using environmental or video sensors. Moreover, such sensors are inexpensive and unobtrusive, and do not raise privacy concerns. Combining multisensor systems with wearable devices can also prevent false or missed alarms as it analyzes the full range of user activity [14].

Accelerometers, tri-axial accelerometers or gyroscopes are the most commonly wearable devices used for fall detection. An accelerometer is used to detect the magnitude and direction of the acceleration along a single axis or multiples axes [15]. Analysis of accelerometer outputs allows for the detection of specific events based on fall detection algorithms. Accelerometers 


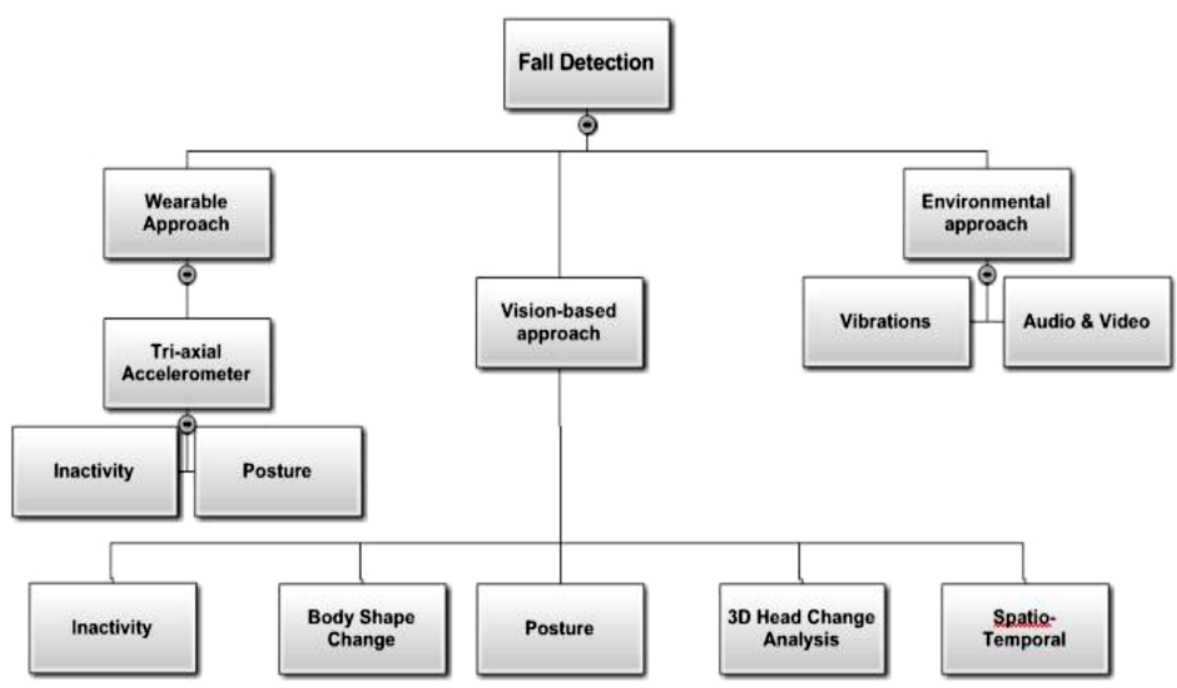

Figure 1. Fall detection classification.

have been used to facilitate long-term monitoring using wearable sensor units. Through its embedded core, the accelerometer can reliably detect falls. Moreover, enhancements in micro-electromechanical systems (MEMS) technologies, have significantly reduced the size and cost of accelerometers. In addition, accelerometers have low power consumption and good performance in terms of power consumption and calculation capabilities [16].

\section{Design of a fall detection system}

\section{Requirements}

- Automation: Health monitoring systems must automatically trigger an emergency alarm, since after a fall the patient may be unable to ask for help.

- Reactivity: Prompt fall detection can be a matter of life and death, with quick response reducing risk of serious injuries or mortality.

- Accuracy: System accuracy must be optimized for detecting real falls as well as filtering false alarms. Two criteria of quality can be defined: sensitivity and specificity. Sensitivity (SE) is the capacity to detect real falls (Eq. 1) and is defined as the ratio between the number of falls properly detected (true positives) and falls that actually occurred (true positives plus false negatives).

$$
S E=\frac{T P}{T P+F N}
$$

- Specificity (SP) is the capacity to filter false alarms and corresponds to the ratio between falls properly discarded (true negatives) and the total number of discarded actions ( true and false negatives) (Eq. 2).

$$
S E=\frac{T P}{T P+F N}
$$

The highest degree of accuracy is reached when both sensitivity and specificity values are equal to 1 (or $100 \%)$ [15], meaning that the system always detects real falls and never generates false alarms.

- Privacy: the monitoring device should be almost invisible to patients and should not be a threat to their privacy.

\section{Challenges in designing fall detectors}

The main goal in designing a fall detection system is to prevent the greatest number of fall events and ensure the subject's safety in daily life. Thus fall detectors must address the following challenges [18].

- Performance under real-life conditions: The fall detection system has to be as accurate and reliable as possible, providing high robustness, high sensitivity and specificity.

- Usability: A health-monitoring device should be easy to use as such devices are intended to be used by elderly and disabled people.

- Acceptance: The new technology has to be accepted, practicable by the monitored patient and operate automatically without any intervention.

- Privacy concerns: the protection of patient's personal data must be guaranteed.

\section{General view of a fall detection system}

A fall detection system consists a set of components and can be described by a block diagram as shown in Fig. 2 .

In Fig.2, the core of the fall detection system is the FPGA that controls the sensors (gyroscopes, accelerometers, magnetometer, etc), reads the digital outputs and determines whether an alert is needed in response to a fall.

In addition, a feature extraction and classification 


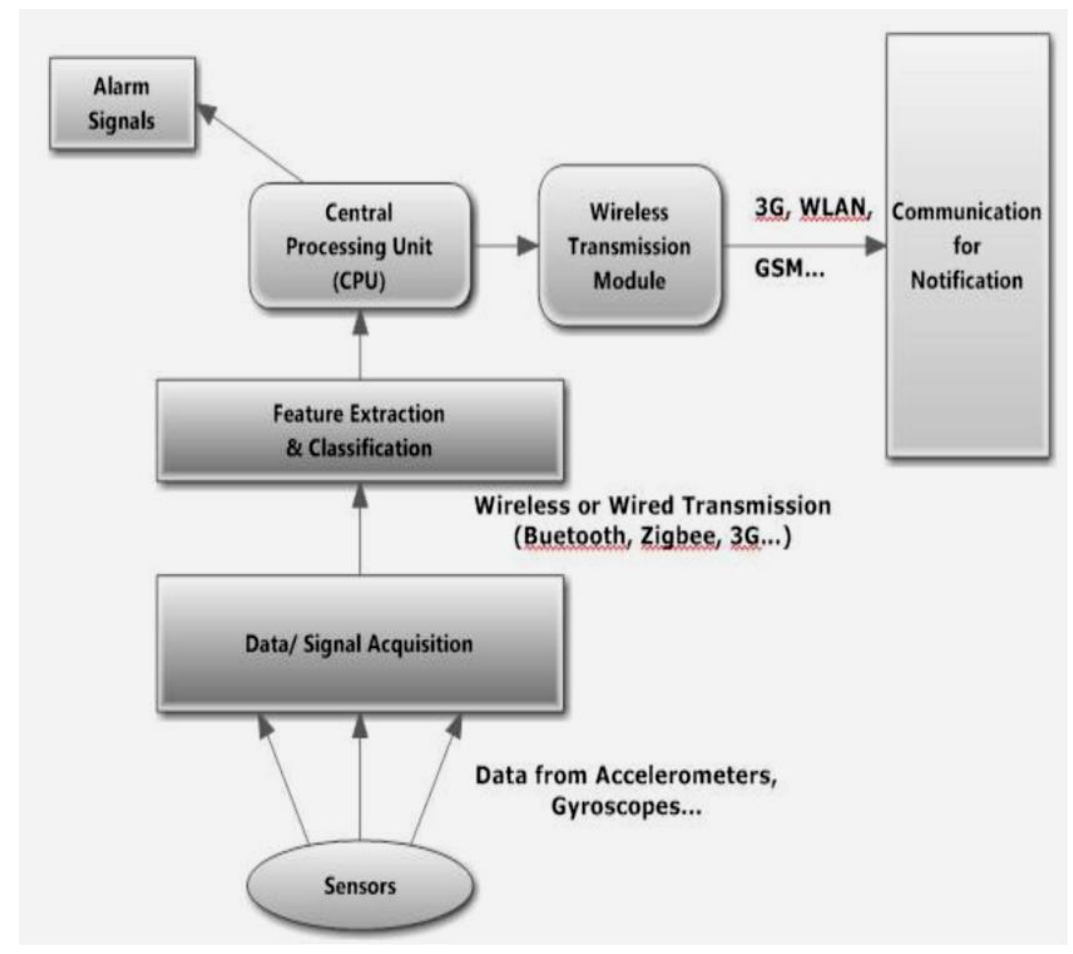

Figure.2. Block diagram of a fall detection system.

algorithm is implemented for data acquisition and analysis.

As wired connections may hinder user movement or present additional risk for falls, sensor data can be transmitted using wireless connections. Long-range communication between the control unit and other stakeholders (e.g., physician, family members) can be handled by a wide range of wireless technologies like WLAN, GSM, 3G, and even 4G.

In terms of hardware requirements, the embedded medical device should provide flexibility and circuit customizability to support real-time, adaptive and multiparameter medical monitoring. A flexible system can be dynamically reconfigured to meet specific application needs, provides high speed throughput, low energy consumption and small surface area for circuit customization needs.

\section{Wireless transmission in fall detection systems}

Wireless communications components are energy hungry [14] and data can be transmitted either by wired or by wireless links.

A wired connection can severely impact the user's mobility and comfort, and raises the risk of system failure. Using a smaller and less power hungry monitoring device allows for obtaining real-time health information from wearable sensors.

Wireless technologies including Zigbee, Bluetooth and WLAN offer higher bandwidth but are more expensive and power hungry especially. In particular, Bluetooth allows only a limited number of communication nodes at a given time [14]. ZigBee offers numerous advantages including low cost, low power consumption, high portability, high reliability and a more extensive range [2].

\section{Fall detection systems using FPGAs}

\section{Advantages of FPGAs in fall detection systems}

Most existing fall detection solutions were developed using either microcontrollers [21] [22] or FPGAs [23] [24] as a CPU of the system.

Using a microcontroller as a processing unit provides improved intelligence, compactness and reliability but is restricted to research of software architectures and applications [14]. Designing hardware architectures and solutions requires the use of programmable logic (FPGA) in order to maximize efficiency and improve calculation capacities.

Using a microcontroller as a processing unit provides improved intelligence compactness and reliability but is restricted to research of software architectures and applications 14]. In addition, the limited performance of low-power microcontrollers makes them unusable, and integrating a more powerful processor will increase power consumption [25] which is the main problem of FPGAs, with the leakage current of highdensity FPGA devices presenting a significant obstacle to battery life because the amount of critical logic 
Table 1. Comparison between FPGAs and microcontrollers in fall detection systems.

\begin{tabular}{|c|c|c|}
\hline$A \&$ & trollers $(\mu C)$ & FPGAs \\
\hline Strengths & $\begin{array}{l}\text { - Shorter set up time and less complex } \\
\text { implementation. } \\
\text { - Proposed solutions using } \mu \mathrm{C} \text { are less } \\
\text { expensive. } \\
\text { - Lower power consumption }\end{array}$ & 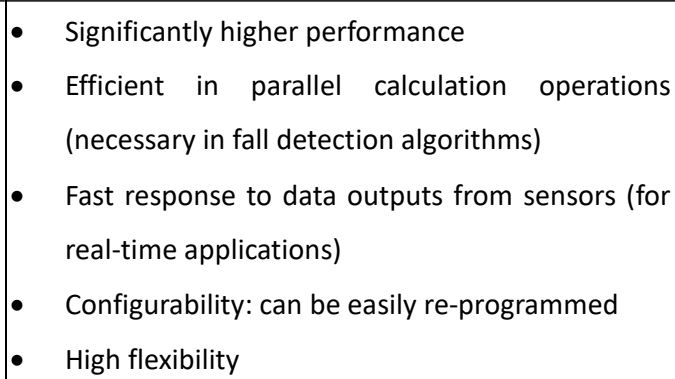 \\
\hline Weakr & $\begin{array}{l}\text { - Performance degrades with increasing } \\
\text { complexity } \\
\text { - Slower response to simultaneous inputs } \\
\text { - Sequential execution }\end{array}$ & $\begin{array}{l}\text { - } \quad \text { Power hungry due to the large number of logic } \\
\text { gates implemented in the board. } \\
\text { - High complexity } \\
\text { - Building devices with FPGAs is more costly. }\end{array}$ \\
\hline
\end{tabular}

implemented in the device requires the device to always be active.

Combined with a processor, FPGA technology can offer a reconfigurable and flexible architecture in a single chip which sidesteps the power consumption problem [10], especially through the recent development of new low-power FPGA devices like LATTICE Semiconductor's iCE40 UltraLite, designed for use in wearable applications, Xilinx's 7 series FPGAs with power estimation and optimization features, and MicroSemi's Flash-based SmartFusion2 SoC and IGLOO2 FPGAs.

Low power microcontrollers can't provide processing capacity required for real-time multiparameter analysis of biomedical signals [26]. FPGA supports fast, real-time and multidimensional signal analysis using statistical techniques and signal processing algorithms which is very useful for biomedical patient monitoring devices [4]. Some systems combining fall detection applications and EEG signal analysis require signal processing speeds of up to $100 \mathrm{MHz}$.

FPGA allows for quick and efficient transmission of raw data acquired from the accelerometer. For fall detection, the memory space available in FPGAs offers a huge advantage over microcontrollers for data sample storage.

FPGA also offers a flexible platform that enables the designer to optimize performance from hardware acceleration [27]. FPGAs provide high integration density and flexibility which makes it possible to implement different on-chip applications like fall-detection systems [28].

The growing use of FPGAs in medical applications has driven the development of solutions to improve the efficiency of floating point (FP) calculations. Xilinx 7 series FPGAs are designed to perform FP operations in a single device using the Xilinx Generator DSP that enables the design and implementation of FP algorithms [50]. Stratix
V FPGAs from Altera [51] are also designed for optimal double precision FP operations and implementation results showed impressive performance with parallelization of different computational elements. OpenCL can also be used to maximize complex FP processing in FPGAs by using the "Fused Datapath" technique [52] where FP processing reduces the number of barrel shifting circuits required, allowing for large scale and high performance FP designs to be built using FPGAs.

FPGAs can be promptly re-configured with an appropriate soft-core processor and a set of suitable peripheral interfaces thus increasing the overall fall detection efficiency and reliability. But to achieve higher performance while meeting critical timing margins using FPGAs, one consistently problematic performance bottleneck is the memory interface. Today's more advanced FPGAs provide embedded blocks in every $1 / 0$ that increase the simplicity and reliability of the interface design. These I/O elements are building blocks that, when combined with surrounding logic, can provide the designer with a complete memory interface controller. It is also possible now with the new FPGA boards to perform a short and efficient data transmission between numerous devices such as sensors, memory and LCD displays through the $12 \mathrm{C}$ bus or SPI communication protocol. The provided interface between the sensor and FPGA is reliable and supports high speed transmission rates.

Thus FPGA presents a promising technology to control accelerometers in fall detection applications. Existing fall detection systems using PFGAs as a CPU

Several recent attempts have been made to use FPGAs as a CPU to address fall detection problems.

Ahola et al. [14] developed a new sensor platform based on an FPGA for various applications including fall detection. The main goal of the proposed advanced sensor processor architecture is to minimize power consumption by performing signal processing on a 
compact and energy processor unit. The hardware prototype was designed to be semi-modular and includes FPGA, battery management and several sensors (i.e., 3axis accelerometer, gyroscope, and magnetometer). As a result, similar to other sensor platforms, to proposed platform is also power hungry with $50 \mathrm{~mA}$ of current consumption in sleep mode and more than $100 \mathrm{~mA}$ in active mode. However, the proposed platform provides a high degree of flexibility through the use of FPGA, allowing for adaptation to a wide range of research areas.

In [23], a wearable wireless accelerometer device based on FPGA was introduced for fall detection in ambient assisted living ( $A A L$ ) applications. Its functions include accelerometer control, reading digital output and delivering the necessary information to the ZigBee radio module. The algorithm proposed here follows a main rule: every time a fall occurs, the acceleration changes significantly to result with the patient assuming a prone, horizontal position in such a way that significantly changes the accelerometer's orientation with respect to gravity.

Other searches [27] have run FPGA in two parallel algorithms to detect falls, where the first algorithm estimates the patient's orientation and the second estimates his acceleration. Findings showed that, on a a dual-core processor, the FPGA implementation offers a great speedup over the software implementation with reduced power consumption.

Combining the vision and wearable approaches, a group of Italian researchers [29] presented a system that integrates two devices: a MEMS wearable wireless accelerometer and a 3D Time-of-Flight camera to detect falls in a home context. Fall detection algorithms are implemented on a low power FPGA, which runs six different parallel routines, with two approaches for each axis. The first measures the stress in terms of fall energy, while the second checks the acceleration shape and the third estimates the orientation of the person after a fall.

The authors in [30] describe the implementation and acceleration of an automatic real-time fall detection system using a Shimmer device. This device is based on compressive sensing reconstruction, orientation and acceleration estimation of data from three axial accelerometers, to produce a fall detection decision. The processor is used to manage and control the different operations and also execute software tasks such as decision making.

In [31], the authors designed a networked multisensory system to produce a data fusion approach that enhances the framework robustness while reducing false positives. The developed architecture is a set of three different sensors: a 3D time-of-flight range camera, a wireless wearable MEMS accelerometer and an off-theshelf microphone. These devices are connected with ad- hoc interface circuits to a main host embedded PC that receives and processes the transmitted data using a multithreading approach.

In [24], a group of researchers developed a new computer architecture, implemented on an FPGA: Xilinx Spartan-3E FPGA. They show the benefits of in-sensor processing through 2 examples: fall detection for the elderly and swimming style recognition for professional athletes.

In [32], falls are considered to be an abnormal activity and are detected by comparing the previous set of measurements against a default (i.e., normal) scenario. The system collects daily activity data and develops an activity pattern using ultrasonic range finding combined with a complicated algorithm. The signal processing capability provided by an FPGA processor is needed to reconstitute the patient activity.

Two fall detectors based on bio-inspired algorithms are described in [25]. These systems includes two devices: an embedded video-based one and a wearable accelerometer-based one. Their architecture is extensible and can be integrated into smart environments. These two devices are connected to a specific framework that implements policies aimed at maximizing system reliability and minimizing the false positives.

A bio-inspired stereo vision fall detection system is introduced in [47]. The system design includes two optical detector chips, an FPGA, a digital signal processor (DSP) and a wireless communication module. The optical chips capture video frames. The FPGA creates input data for the DSP by calculating 3D representations of the environment. The DSP is loaded with a neural network used for classification purposes. Falls are divided into 4 phases: pre-fall, critical, post-fall and recovery phase. Trial results showed a $90 \%$ fall detection rate for all networks, and 97\%-98\% for the best network.

The authors in [48] present the design, simulation and implementation of a novel fall detection system based on the Shimmer platform and Zynq board. In this prototype, accelerometer data are sent to the processing unit by Bluetooth. The Discrete Wavelet Transform (DWT) is used to preprocess the raw data collected from the Shimmer platform. Dimensionality reduction is implemented using Principal Component Analysis (PCA) and finally classification is carried out using a Decision Tree (DT) classifier. This system has been designed and tested using MATLAB for software verification and then the hardware implementation has been performed on Zynq FPGA (SoC). Using the Zynq platform permitted a high degree of flexibility in system design, and a performance can be enhanced depending on the degree of design complexity.

Bianchi et Al [49] introduced integrated a wireless 
Table 2. Comparison of FPGA-based fall detection systems in the literature.

\begin{tabular}{|c|c|c|c|c|c|c|c|c|}
\hline Reference & $\begin{array}{c}\text { Type of } \\
\text { Algorithm }\end{array}$ & CPU & Sensor & $\begin{array}{c}\text { Obstructiv } \\
\text { e }\end{array}$ & $\begin{array}{c}\text { Energy } \\
\text { Consumpti } \\
\text { on }\end{array}$ & Cost & Complexity & Results \\
\hline [14] & $\begin{array}{l}\text { Thresholds } \\
\text { Comparison }\end{array}$ & $\begin{array}{l}\text { Altera } \\
\text { Cyclone II }\end{array}$ & $\begin{array}{l}\text { Accelerome } \\
\text { ter } \\
\text { Gyroscope } \\
\text { Magnetome } \\
\text { ter }\end{array}$ & LOW & HIGH & HIGH & HIGH & $\begin{array}{l}\text { More efficient } \\
\text { in signal } \\
\text { processing } \\
\text { algorithms }\end{array}$ \\
\hline [23] & $\begin{array}{l}\text { Low Th } \\
\text { Medium Th } \\
\text { High Th }\end{array}$ & $\begin{array}{l}\text { FPGA } \\
\text { Spartan III }\end{array}$ & $\begin{array}{l}\text { 3D- } \\
\text { Accelerome } \\
\text { ter }\end{array}$ & HIGH & $\begin{array}{l}\text { LOW } \\
\text { (Xbee Pro) }\end{array}$ & MEDIUM & LOW & $\begin{array}{l}\text { Efficiency } \\
98 \%, \quad 88 \%, \\
55 \% \\
\text { Reliability } \\
61 \%, \quad 80 \% \text {, } \\
97 \%\end{array}$ \\
\hline [27] & $\begin{array}{l}\text { Orientation \& } \\
\text { Acceleration } \\
\text { Estimation }\end{array}$ & $\begin{array}{l}\text { FPGA } \\
\text { Virtex V }\end{array}$ & $\begin{array}{l}\text { Shimmer } \\
\text { Device }\end{array}$ & LOW & HIGH & HIGH & MEDIUM & $\begin{array}{l}\text { Specificity } \\
98 \%, 98 \% \\
\text { Sensitivity } 94 \\
\%, 96 \%\end{array}$ \\
\hline [29] & $\begin{array}{l}\text { Thresholds } \\
\text { Comparison }\end{array}$ & FPGA & $\begin{array}{l}\text { MESA 3D } \\
\text { Tof } \\
\text { Camera } \\
\text { 3D MEMS }\end{array}$ & HIGH & MEDIUM & HIGH & MEDIUM & $\begin{array}{l}\text { Efficiency } \\
88.4 \% \\
\text { Reliability } \\
79.3 \%\end{array}$ \\
\hline [30] & $\begin{array}{l}\text { OMP+ } \\
\text { Thresholds }\end{array}$ & $\begin{array}{l}\text { Zedboard } \\
\text { Zynq } 7000\end{array}$ & $\begin{array}{l}\text { Shimmer } \\
\text { Device }\end{array}$ & MEDIUM & MEDIUM & MEDIUM & HIGH & $\begin{array}{lr}30 \% & \text { reduction } \\
\text { in } & \text { power } \\
\text { consumption }\end{array}$ \\
\hline [31] & $\begin{array}{l}\text { Low Th } \\
\text { Medium Th } \\
\text { High Th }\end{array}$ & $\begin{array}{l}\text { FPGA+ } \\
\text { GNU } \\
\text { Linux } \\
\text { based } \\
\text { e_PC }\end{array}$ & $\begin{array}{l}\text { 3D Camera } \\
\text { Zigbee } \\
\text { Wireless } \\
\text { Accelerome } \\
\text { ter } \\
\text { Microphone }\end{array}$ & HIGH & HIGH & HIGH & MEDIUM & $\begin{array}{l}\text { Efficiency } \\
98 \%, \quad 88.4 \% \text {, } \\
50,1 \% \\
\text { Reliability } \\
56 \%, \quad 79.3 \% \text {, } \\
96.2 \%\end{array}$ \\
\hline [24] & $\begin{array}{l}\text { SVM } \\
\text { Classifier+ } \\
\text { Threshold }\end{array}$ & $\begin{array}{l}\text { FPGA } \\
\text { Xilinx } \\
\text { S3E1200 }\end{array}$ & $\begin{array}{l}\text { Wireless } \\
\text { 3D- } \\
\text { Accelerome } \\
\text { ter } \\
\text { Vision } \\
\text { Sensor } \\
\text { Node } \\
\end{array}$ & LOW & HIGH & HIGH & HIGH & $\begin{array}{l}\text { Efficiency } \\
99.05 \% \\
\text { Reliability } \\
97.05 \%\end{array}$ \\
\hline [32] & $\begin{array}{l}\text { Initialization } \\
\& \quad \text { Decision } \\
\text { Making } \\
\text { Procedure }\end{array}$ & $\begin{array}{l}\text { FPGA+ } \\
\text { Netbook }\end{array}$ & $\begin{array}{l}\text { Ultrasonic } \\
\text { sensors }\end{array}$ & MEDIUM & HIGH & HIGH & HIGH & Still in testing \\
\hline [25] & $\begin{array}{l}\text { SMA \& SVM } \\
\text { Classifer + } \\
\text { Threshold } \\
\text { Comparison }\end{array}$ & $\begin{array}{l}\text { Spartan 3E } \\
1200 \\
\text { FPGA }\end{array}$ & $\begin{array}{l}\text { 3D- } \\
\text { Accelerome } \\
\text { ter } \\
\text { Freescale } \\
\text { MMA } \\
7260 Q \\
\end{array}$ & LOW & MEDIUM & MEDIUM & HIGH & $\begin{array}{l}\text { Accuracy } \\
91.24 \%\end{array}$ \\
\hline [49] & $\begin{array}{l}\text { Threshold } \\
\text { and body tilt } \\
\text { comparison }\end{array}$ & $\begin{array}{l}\text { Xilinx } \\
\text { Spartan } \\
\text { XC3S500 } \\
\text { E }\end{array}$ & $\begin{array}{l}\text { 3D MEMS } \\
\text { Accelerome } \\
\text { ter }\end{array}$ & LOW & MEDIUM & MEDIUM & LOW & $\begin{array}{l}40 \% \text { reduction } \\
\text { in power } \\
\text { consumption }\end{array}$ \\
\hline [48] & $\begin{array}{l}\text { DWT } \\
\text { PCA } \\
\text { DT }\end{array}$ & Zynq 7000 & $\begin{array}{l}\text { Shimmer } \\
\text { Device }\end{array}$ & MEDIUM & MEDIUM & MEDIUM & HIGH & $\begin{array}{l}\text { Accuracy } \\
88.4 \%\end{array}$ \\
\hline
\end{tabular}

sensor platform to a home control and monitoring network to recognize abnormal gait and falls. Low power strategies based on a threshold fall detection algorithm were implemented at both the software and hardware levels (both for microcontroller and FPGA). The sensor platform prototype includes a 3D accelerometer, a Zigbee transceiver and a PIC18LF4620 microcontroller. Implemented as a subsystem of an ASIC design, this approach showed significant advantages in terms of reduced power consumption, along with increased reliability and portability.

\section{Points to improve in fall detection systems}

The performance of currently available automatic fall detection systems is relatively limited. Fall detection systems in the literature still require significant 
improvement before real-world deployment. Current key disadvantages include:

- False alarms: Current fall detection systems frequently misinterpret certain common activities as falls, including sitting down or jumping [33]. These false positives will trigger alerts, thus decreasing system effectiveness [18].

- Inconvenience: Existing fall detection systems present logistical difficulties for elderly people to use in their daily lives as their components regularly need to be re-charged, and some must be manually activated. These details need to re-worked to ensure ease of use and user autonomy.

- Charging the system: The sleep cycle of sensing components must be carefully managed to minimize battery drain [8], as frequent recharging may present particular challenges for elderly or disabled users. Future studies should reconsider power control issues in fall detection devices to increase system robustness and effectiveness.

- Unreliability: The high frequency of false positives make existing solutions unreliable. Designers cannot expect to subject older people to simulated falls, thus creating difficulties for detector evaluation. In addition, fall causes are not recorded, and devices are only tested for a limited number of scenarios (e.g., lying down, a backward fall, forward fall, etc) [34].

\section{Fall detection algorithms}

\section{Techniques for fall-detection with acceleration-based systems}

A fall is a change in body orientation from standing position to lying. It's a process lasting 1 to 5 seconds consisting of several sub-actions that are analyzed to detect the occurrence of a fall (Fig. 3). A fall is immediately preceded by a large negative acceleration [32]. Falls are also characterized by a violent impact on a hard surfaces causing sudden peaks in the acceleration magnitude's graph [33].

Tests in $[16,35]$ showed that accelerometers placed on the waist and chest give best results for fall detection. Fall detection techniques based on acceleration magnitude and speed of sign change include:

- Threshold-based methods (TBM): a fall is reported when the acceleration peaks or valleys reach predefined thresholds. This is the simplest and most commonly used approach to detect falls, and the alert is automatically triggered when the acceleration threshold value is reached [36,37]. This approach entails lower computational cost but suffers from high rates of false positive and true negative results [38]. This approach provides weak specificity values because some impacts can be caused by ordinary living activities.

- Machine learning methods (MLM) are based on dynamic activity recognition and their rules are not yet sufficiently accurate [39]. This method is a representation of individual behavior and a classification of different activities to detect a fall among other movements. The user's behavior is first classified into different activities. These activities are recorded and split into 1s fragments. Each activity is assigned to an attributed vector which represents 10 consecutive snapshots of the user's posture. The attribute vectors are used as training data for each MLM algorithm. This method shows better adaptability to different subjects because human movements are considered as processes instead of isolated points, but training requires a wide range of isolated points from reallife scenarios [40].

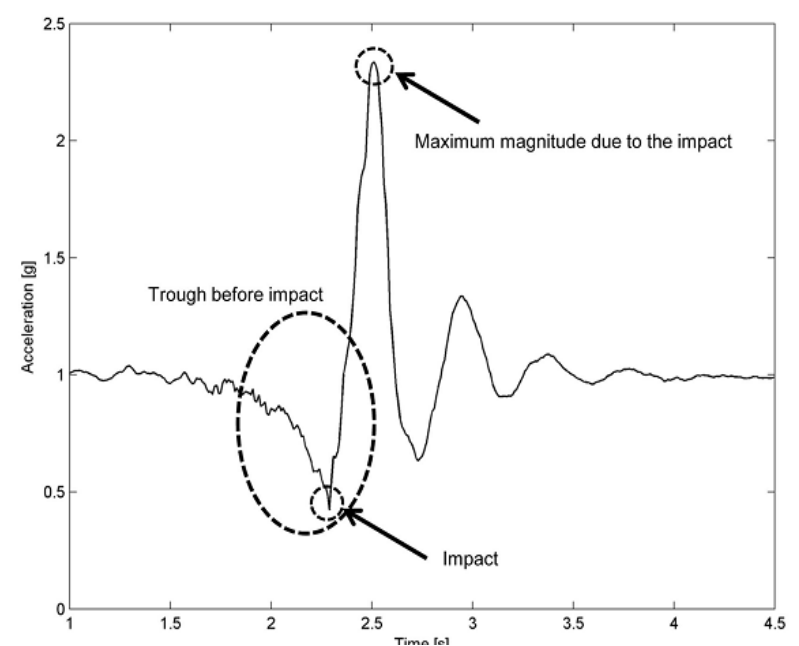

Figure 3. Prototypical acceleration sum vector of a fall

\section{Mostly used algorithms}

\section{Threshold-based Algorithms}

Threshold-based algorithms check if a person's acceleration is above a fixed value. Impact detection is calculated by following TBM. Threshold-based algorithms have low computational cost and complexity making them easy to implement for real-time applications.

Previous studies $[1,41,42]$ have tested thresholdbased algorithms with Bourke [43] showing the best results including a reduction of number of features, low computational cost and easy integration in embedded solutions. However, such approaches are subject to limitations including low sensitivity for soft-falls (i.e., falls against a wall), and for break-falls when a person acts to reduce the fall impact [44]. Thresholds used in most studies are calibrated on simulated false signals and are not suitable for detection of real-world falls [45]. 


\section{SVM Algorithms}

SVM is a robust classification tool with good generalizability [34]. It divides all samples into objective \& non-objective fields, which are then mapped into a higher dimensional feature spaces with efficient operators, called kernel functions [38]. The most commonly used fall parameters in SVM are ASVM: SVM of acceleration and $\theta$ (i) (the angle between the accelerometer $y$-axis and the vertical direction).

$$
\begin{aligned}
& \mathrm{A}_{\mathrm{SVM}}(\mathrm{i})=\sqrt{\left(A_{x}^{2}(i)+A_{y}^{2}(i)+A_{z}^{2}(i)\right)} \\
& \theta(\mathrm{i})=\tan ^{-1}\left(\frac{\sqrt{\left(A_{y}^{2}(i)+A_{Z}^{2}(i)\right)}}{A_{x}(i)}\right) \times \frac{180}{\pi}
\end{aligned}
$$

where $A x(i), A y(i)$ and $A z(i)$ respectively correspond to the $x$-axial, $y$-axial and z-axial accelerations of the ith sample.

SVM is often chosen for its high performance in binary classification tasks with small number of samples [40]. The SVM algorithm is based on training, testing and performance evaluation, which are common steps in every learning procedure. Training involves optimization of a convex cost function where there are no local minima to complicate the learning process. Testing is based on the model evaluation using the support vectors to classify a test data set [46].

The OC-SVM algorithm shows better results of specificity and sensitivity while respecting the threshold based approach, detecting low-impact falls at a higher computational cost since the number of features is compact and can be integrated in low-cost embedded solutions (DSP, FPGA, $\mu$ C) [16].

It is very important for the designer to consider all application features to select the proper fall detection algorithm as it determines system reliability and efficiency.

\section{FPGA interfaces to FPGA communication interfaces}

Several studies have attempted to detect falls among elderly subjects using accelerometers, with the main steps for fall detection described in Fig. 4.

The motion data pattern of a fall is distinct from that of other activities. Therefore, a fall detection algorithm must be developed that calculates the acceleration from the data collected by the 3-axis accelerometer. In fact, developing an appropriate fall detection algorithm is a key design step for developing an efficient system as early response is critical for reducing serious injury. The triaccelerometer data are acquired and analyzed through FPGAs interfaces (SPI, 12C...) and the fall detection decision technique is implemented on the FPGA. When a fall occurs, the system directly sends an alert to family

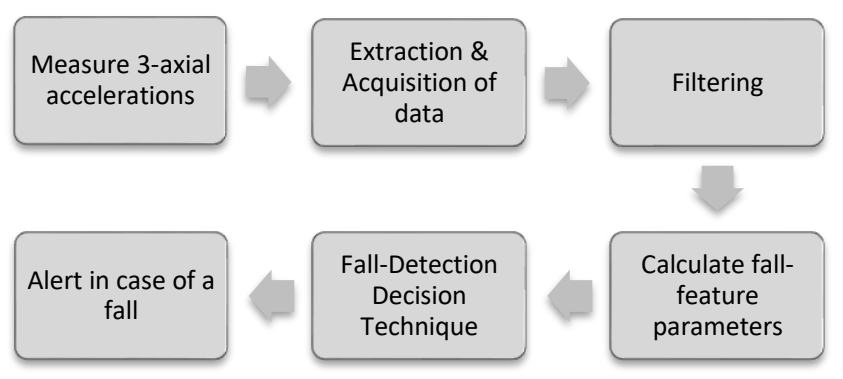

members or caregivers through $\mathrm{Wi}-\mathrm{Fi}, 3 \mathrm{G}$. or other wireless technologies. Alerts can also be displayed using LEDs on the FPGA board.

Figure 4. Logical framework overview.

\section{Conclusion}

We present a survey of fall detection systems including approaches and definitions focusing on systems using FPGAs as a CPU. We provide an overview of fall detection systems using accelerometers, along with descriptions and comparisons of fall detection systems using FPGAs, identifying their relative advantages and shortcomings, while describing the main fall detection techniques and algorithms. The results of the survey show that most fall detection systems are tested for simulated falls, but are not particularly well-suited for use in real-life scenarios. We also suggest that a framework for fall detection should adopt a modular approach that takes into consideration standards for interoperability, security, privacy, multiple hardware platforms, wireless communications protocols and multiple application interfaces.

\section{Acknowledgements}

This research and innovation work is carried out within a MOBIDOC scholarship funded by the EU under the PASRI project.

\section{References}

[1] J. Chen, K. Kwong, D. Chang, J. Luk, and R. Bajcsy, "Wearable Sensors for Reliable Fall Detection," in proceeding of the 2005 IEEE Engineering in Medicine and Biology 27th Annual Conference, Shanghai, China, Sep. 1-4, 2005, pp. 3551-3354. doi: $\underline{\text { 10.1109/IEMBS.2005.1617246 }}$

[2] M. A. E. Khaddar, H. Harroud, M. Boulmalf, M. Elkoutbi, A. Habbanil, "Emerging Wireless Technologies in E-Health: Trends, Challenges, and Framework Design Issues," in proceeding of 
Multimedia Computing and Systems International Conference, Tangier, Morocco, May 10-12, 2012, pp. 440-445.

doi: $10.1109 /$ ICMCS.2012.6320276

[3] A. Pantelopoulos and N. G. Bourbakis, "A Survey on Wearable Sensor-Based Systems for Health Monitoring and Prognosis," IEEE Transactions on Systems, Man, and Cybernetics Part C: Applications and Reviews, vol. 40, no. 1, pp. 1-12, 2010. doi: 10.1109/TSMCC.2009.2032660

[4] D. M. Karantonis, M. R. Narayanan, M. Mathie, N. H. Lovell, and B. G. Celler, "Implementation of a RealTime Human Movement Classifier Using a Triaxial Accelerometer for Ambulatory Monitoring," IEEE Transactions on Information Technology in Biomedicine, vol. 10, no. 1, pp. 156-167, 2006. doi: 10.1109/TITB.2005.856864

[5] P. Ong, C. Ooi, Y.-C. Chang, E. K. Karuppiah, and S. M. Tahir, "An FPGA-Based Hardware Implementation of Visual based Fall Detection," in proceeding of 2014 IEEE Region 10 Symposium, Kuala Lumpur, Malaysia, April 14-16, 2014, pp. 397-402. doi: 10.1109/TENCONSpring.2014.6863065

[6] Yi. He, Ye. Li, and S.-O. Bao, "Fall Detection by BuiltIn Tri-Accelerometer of Smartphone," in proceeding of IEEE-EMBS International Conference on Biomedical and Health Informatics, Hong Kong, China, Jan. 2-7, 2012, pp. 184-187. doi: $10.1109 / \mathrm{BHI} .2012 .6211540$

[7] P. Leijdekkers, V. Gay, and E. Laurence, "Smart Homecare system for Health Tele-Monitoring," in proceeding of The First International Conference on the Digital Society, Guadeloupe, France, Jan. 2-6 2007, p. 3. doi: $10.1109 /$ ICDS.2007.37

[8] M. Mubashir, L. Shao, and L. Seed, "A survey on fall detection: Principles and approaches," Elsevier Neurocomputing, vol.100, pp. 144-152, 2013. doi: 10.1016/i.neucom.2011.09.037

[9] R. Hegde, B. G. Sudarshan, S. C. Kumar, H. S. Ac, and B. S. Satyanarayana, "Technical Advances in Fall Detection System - A Review," International Journal of Computer Science and Mobile Computing, vol. 2, no. 7, pp. 152-160, 2013.

[10] C. Hu, Z. Liu, Y. Pan, and Z. Zeng, "A Novel FPGABased Wireless Vision Sensor Node," in proceeding of IEEE International Conference on Automation and Logistics, Shenyang, China, Aug. 5-7, 2009, pp. 841846.

doi: $10.1109 /$ ICAL.2009.5262805

[11] Z. Pang, Q. Chen, and L. Zheng, "A Pervasive and Preventive Healthcare Solution for Medication Noncompliance and Daily Monitoring," in proceeding of Applied Sciences in Biomedical and Communication Technologies, Bratislava, Slovakia, Nov. 24-27, 2009, pp. 1-6.

doi: 10.1109/ISABEL.2009.5373681

[12] E. Jovanov, E. Wang, L. Verhagen, M. Fredrickson, and R. Fratangelo, "deFOG - a Real Time System for detection and unfreezing of Gait of Parkinson's Patients," in proceeding of Annual International Conference of the IEEE, Minneapolis, MN, Sep. 3-6, 2009, pp. 5151-5154. doi: $10.1109 /$ IEMBS.2009.5334257

[13] J. Dai, X. Bai, Z. Yang, Z. Shen, and D. Xuan, “PerFallD: A Pervasive Fall Detection System Using Mobile Phones," in proceeding of Pervasive Computing and Communications Workshops, Mannheim, Germany, Mar. 29-Apr. 2, 2010, pp. 292-297. doi: 10.1109/PERCOMW.2010.5470652

[14] T. Ahola, P. Korpinen, J. Rakkola, T. Ramo, J. Salminen, and J. Savolainen, "Wearable FPGA Based Wireless Sensor Platform," in proceeding of the 29th Annual International Conference of the IEEE EMBS, Lyon, France, Aug. 22-26, 2007, pp. 2288-2291. doi: $10.1109 /$ IEMBS.2007.4352782

[15] A. M. Khan, "Human Activity Recognition Using A Single Tri-axial Accelerometer," Ph.D. dissertation, Department of Computer Engineering, Kyung Hee University, Seoul, Korea, 2011.

[16] W.-C. Cheng and D.-M. Jhan, "Triaxial Accelerometer-Based Fall Detection Method Using a Self-Constructing Cascade-AdaBoost-SVM Classifier," IEEE Journal OF Biomedical and Health Informatics, vol. 17, no. 2, pp. 411-419, 2013. doi: 10.1109/JBHI.2012.2237034

[17] N. Jia, "Detecting Human Falls with a 3-Axis Digital Accelerometer," Analog Dialogue, vol. 43, no.7, pp. 1-7, 2009. doi: $10.1109 /$ IEMBS.2007.4352355

[18] R. Igual, C. Medrano and I. Plaza, "Challenges, issues and trends in fall detection systems," BioMedical Engineering OnLine, vol. 12, no. 1, pp. 66-90, 2013. doi: $10.1186 / 1475-925 X-12-66$

[19] H. Yu and L. Liu, "Remote Health Monitoring System Using ZigBee Network and GPRS Transmission Technology," in proceeding of International Symposium Computational Intelligence and Design (ISCID), Hangzhou, China, Oct. 28-30, 2011, pp. 152 153.

doi: $10.1109 /$ ISCID.2011.46

[20] D. J. R. K. Kumar and N. Kotnana, "Design and Implementation of Portable Health Monitoring system using PSoC Mixed Signal Array chip," International Journal of Recent Technology and Engineering, vol.1, no.3, pp. 2277-3878, 2012. 
[21] Q. Li, J. F. A. Stankovic, M. Hanson, A. Barth and J. Lach, "Accurate, Fast Fall Detection Using Gyroscopes and Accelerometer-Derived Posture Information," in proceeding of Wearable and Implantable Body Sensor Networks, Berkeley, CA, June 3-5, 2009, pp. 138-143. doi: 10.1109/BSN.2009.46

[22] P. Pivato, S. Dalpez, D. Macii, and D. Petri, "A Wearable Wireless Sensor Node for Body Fall Detection," in proceeding of IEEE International Workshop on Measuring and Networking, Ana Capri, Italy, Oct. 10-11, 2011, pp. 116-121. doi: 10.1109/IWMN.2011.6088492

[23] A. Lombardi, M. Ferri, G. Rescio, M. Grassi, and P. Malcovati, "Wearable Wireless Accelerometer with Embedded Fall-Detection Logic for Multi-Sensor Ambient Assisted Living Applications," in proceeding of IEEE SENSORS, Christchurch, New Zealand, Oct. 25-28, 2009, pp. 1967-1970. doi: $10.1109 /$ ICSENS.2009.5398327

[24] S. Cagnoni, G. Matrella, M. Mordonini, F. Sassi, and L. Ascari, "Sensor Fusion-oriented Fall Detection for Assistive Technologies Applications," in proceeding of IEEE 9th International Conference on Intelligent Systems Design and Applications, Pisa, Italy, Nov. 30Dec. 2, 2009, pp. 673-678. doi: $10.1109 /$ ISDA.2009.203

[25] B. Pfundt, M. Reichenbach, B. Eskofier, and D. Fey, "Smart Sensor Architectures for Embedded Biosignal Analysis," in proceeding of Design and Architectures for Signal and Image Processing (DASIP), Cagliari, Italy, Oct. 8-10, 2013, pp. 174-181.

[26] H. Alemzadeh, Z. Jin, Z. Kalbarczyk, and R. K. Iyer, "An Embedded Reconfigurable Architecture for Patient-Specific Multi-Parameter Medical Monitoring," in proceeding of IEEE 2011 Annual International Conference in Engineering in Medicine and Biology Society, Boston, MA, Aug. 30-Sep. 3, 2011, pp. 1896-1900. doi: 10.1109/IEMBS.2011.6090537

[27] M. Neggazi, A. Amira, and L. Hamami, "A Wireless Reconfigurable System for Falls Detection," in proceeding of IEEE 11th International Conference on Information Sciences, Signal Processing and their Applications, Montreal, QC, July 2-5, 2012, pp. 7782.

doi: $10.1109 /$ ISSPA.2012.6310658

[28] C. Chou, W. Fang, and H. Huang, "A Novel Wireless Biomedical Monitoring System with Dedicated FPGA-based ECG Processor," in proceeding of IEEE 16th International Symposiumon Consumer Electronics (ISCE), Harrisburg, PA, June. 4-6, 2012, pp.1-4. doi: 10.1109/ISCE.2012.6241744

[29] M. Grassi, A. Lombardi, G. Rescio, M. Ferri, P. Malcovati, A. Leone, G. Diraco, P. Siciliano, M. Malfatti, and L. Gonzo, "An Integrated System for People Fall-Detection with Data Fusion Capabilities Based on 3D ToF Camera and Wireless Accelerometer," in proceeding of IEEE SENSORS 2010 Conference, Kona, HI, Nov. 1-4, 2010, pp.10161019.

doi: 10.1109/ICSENS.2010.5690746

[30] H. Rabah, A. Amira, and A. Ahmad, "Design and Implementation of a Fall Detection System using Compressive Sensing and Shimmer Technology," in proceeding of 24th International Conference on Microelectronics (ICM), Algiers, Algeria, Dec. 16-20, 2012, pp. 1-4.

doi: $10.1109 /$ ICM.2012.6471399

[31] M. Grassi, A. Lombardi, G. Rescio, P. Malcovati, A. Leone, G. Diraco, C. Distante, and P. Siciliano, "A Hardware-Software Framework for High-Reliability People Fall Detection," in proceeding of IEEE SENSORS 2008 Conference, Lecce, Italy, Oct. 26-29, 2008, pp. 1328-1331. doi: 10.1109/ICSENS.2008.4716690

[32] Y. Huang and K. Newman, "Improve Quality of Care with Remote Activity and Fall Detection Using Ultrasonic Sensors," in proceeding of 34th Annual International Conference of the IEEE Engineering in Medicine and Biology Society, San Diego, CA, Aug. 28-Sep. 1, 2012, pp. 5854-5857. doi: 10.1109/EMBC.2012.6347325

[33] S. Abbate, M. Avvenuti, G. Cola, P. Corsini, J. Light, and A. Vecchio, "Recognition of false alarms in fall detection systems," in proceeding of IEEE Consumer Communications and Networking Conference, Las Vegas, NV, Jan. 9-12, 2011, pp. 23-28. doi: 10.1109/CCNC.2011.5766464

[34] G. Rescio, A. Leone, and P. Siciliano, "Supervised Expert System for Wearable MEMS AccelerometerBased Fall Detector," Journal of Sensors, vol. 2013, no. 2013, pp.1-11.

doi: $10.1155 / 2013 / 254629$

[35] H. Gjoreski, M. Luštrek, and M. Gams, "Accelerometer Placement for Posture Recognition and Fall Detection," in proceeding of IEEE 7th International Conference on Intelligent Environments, Nottingham, UK, July 25-28, 2011, pp. 47-54. doi: 10.1109/IE.2011.11

[36] M. Kangas, A. Konttila, I. Winblad, and T. Jämsä, "Determination of simple thresholds for accelerometry-basedparameters for fall detection," in proceeding of the 29th Annual International 
Conference of the IEEE Engineering in Medicine and Biology Society, Lyon, France, Aug. 23-26, 2007, pp. 1367-1370.

doi: $10.1109 /$ IEMBS.2007.4352552

[37] G. Wu and S. Xue, "Portable Preimpact Fall Detector With Inertial Sensors," IEEE Transactions on neural systems and Rehabilitation Engineering, vol. 16, no. 2, pp. 178-182, 2008. doi: $10.1109 /$ TNSRE.2007.916282

[38] A. Leone, G. Rescio, and P. Siciliano, "Supervised Wearable Wireless System for Fall Detection," in proceeding of IEEE International Workshop on Measurements and Networking, Naples, Italy, Oct. 7-8, 2013, pp. 500-505. doi: 10.1109/IWMN.2013.6663803

[39] H. Gjoreski, S. Kozina, M. Gams, and M. Luštrek, "RAReFall - Real-time Activity Recognition and Fall Detection System," in proceeding of 2014 IEEE International Conference on Pervasive Computing and Communications Demonstrations, Budapest, Hungary, March 24-28, 2014, pp. 145-147. doi: 10.1109/PerComW.2014.6815182

[40] S. Shan and T. Yuan, "A Wearable Pre-Impact Fall Detector using Feature Selection and Support Vector Machine," in proceeding of IEEE 10th International Conference on Signal Processing, Beijing, China, Oct. 24-28, 2010, pp. 1686-1689. doi: 10.1109/ICOSP.2010.5656840

[41] A. K. Bourke, P. van de Ven, M. Gamble, R. O'Connor, K. Murphy, E. Bogan, E. McQuade, P. Finucane, G. ÓLaighin, and J. Nelson, "Assessment of Waist-worn Tri-axial Accelerometer Based Falldetection Algorithms using Continuous Unsupervised Activities," in proceeding of Annual International Conference of the IEEE on Engineering in Medicine and Biology Society, Buenos Aires, Argentina, Aug. 31-Sep. 4, 2010, pp. 2782-2785. doi: 10.1109/IEMBS.2010.5626364

[42] M. Kangas, A. Konttila, P. Lindgren, I. Winblad, T. Jamsa, "Comparison of low-complexity fall detection algorithms for body attached accelerometers," Gait \& posture, vol. 28, no. 2, pp. 285-291, 2008. doi:DOI: 10.1016/j.gaitpost.2008.01.003

[43] A. K. Bourke, J. V. O'Brien, and G. M. Lyons, "Evaluation of a threshold-based tri-axial accelerometer fall detection algorithm," Gait \& posture, vol.26, no. 2, pp.194-199, 2007. doi: 10.1016/i.gaitpost.2006.09.012

[44] Yuwono, Mitchell, et al. "Unsupervised machinelearning method for improving the performance of ambulatory fall-detection systems," Biomedical engineering online, vol. 11, no. 1, pp. 1-9, 2012. doi: $10.1186 / 1475-925 X-11-9$

[45] F. Bagalà, C. Becker, A. Cappello, L. Chiari, K. Aminian, J. M. Hausdorff, W. Zijlstra, and J. Klenk, "Evaluation of Accelerometer-Based Fall Detection Algorithms on Real-World Falls," PloS one, vol.7, no. 5, 2012. doi: 10.1371/journal.pone.0037062

[46] C. Doukas, I. Maglogiannis, F. Tragkas, D. Liapis, and G. Yovanof, "Patient Fall Detection using Support Vector Machines," in proceeding of IFIP International Conference on Artificial Intelligence Applications and Innovations, Athens, Greece, Sep. 23-25, 2007, pp. 147-156. doi: $10.1007 / 978-0-387-74161-116$

[47] A. Belbachir, M. Litzenberger, S. Schraml, M. Hofstatter, D. Bauer, P. Schon, M. Humenberger, C. Sulzbachner, T. Lunden, T. M. Merne, "CARE: A dynamic stereo vision sensor system for fall detection," in proceeding of the International Symposium on Circuits and Systems, Seoul, Korea, May 20-23, 2012, pp. 731-734.

[48] A. A. S. Ali, M. Siupik, A. Amira, F. Bensaali, and P. C.de-la-Higuera "HLS Based Hardware Acceleration on the Zynq SoC: a case study for fall detection system," in proceeding of IEEE 11th International Conference on Computer Systems and Applications, Doha, Qatar, Nov. $10-13,2014$, pp. 685-690. doi: 10.1109/AICCSA.2014.7073266

[49] V. Bianchi, F. Grossi, G. Matrella, I. De Munari, and P. Ciampolini, "A Wireless Sensor Platform for Assistive Technology Applications," in proceeding of 11th EUROMICRO Conference on Digital System Design Architectures, Method and Tools, Parma, Italy, Sep. 3-5, 2008, pp. 809-816. doi: 10.1109/DSD.2008.131

[50] T. Vanevenhoven, "High-Level Implementation of Bit- and Cycle-Accurate Floating-Point DSP Algorithms with Xilinx FPGAs," White Paper WP409, 2011.

[51] Altera, "Designing and Using FPGAs for DoublePrecision Floating-Point Math," White Paper WP01028-1.1, 2007.

[52] M. Parker, "Optimizing complex floating point calculations on FPGAs," Altera Corp., San José, CA, Embedded Systems Conference, 2014. 